\title{
The Effects of Auditory Short-Term Training in Passive Oddball Paradigm with Novel Stimuli
}

\author{
Woojae Han ${ }^{1}$, Jeonghye Park ${ }^{2}$ and Junghwa Bahng ${ }^{2}$ \\ ${ }^{1}$ Division of Speech Pathology and Audiology, Research Institute of Audiology and Speech Pathology, College of Natural Sciences, \\ Hallym University, Chuncheon, \\ ${ }^{2}$ Department of Audiology, Hallym University of Graduate Studies, Seoul, Korea
}

Received September 26, 2013

Revised November 5, 2013

Accepted November 25, 2013

Address for correspondence
Junghwa Bahng, PhD
Department of Audiology,
Hallym University
of Graduate Studies,
405 Yeoksam-ro, Gangnam-gu,
Seoul 135-841, Korea
Tel +82-70-8680-6933
Fax +82-2-3453-6618
E-mail bahng.jh@gmail.com

Address for correspondence

Hallym University

of Graduate Studies,

E-mail bahng.jh@gmail.com

\begin{abstract}
Background and Objectives: The purpose of this study was to determine how human neural activity might be changed through auditory short-term training when listening to novel stimuli. Subjects and Methods: Among the twenty young normal hearing adult listeners who participated, ten were randomly assigned to a training group and ten were assigned to a nontraining group as a control. Two synthesized novel stimuli were used: / su/ and / Ju/. Both stimuli similarly sounded like /su/, but had two different onset transition frequencies and fricative pole frequencies. In the experiment, behavioral identification test (i.e., /su/ vs. / $u$ /) and the mismatch negativity ( $\mathrm{MMN}$ ) were measured before and after training for the training group. To gauge the training effect, the listeners in the training group were taught by discrimination and identification of two novel stimuli for about 20 minutes. Results: The results showed that scores for the behavioral test increased significantly after auditory short-term training. Also, onset latency, duration, and area of the MMN were significantly changed when elicited by the training stimuli. Conclusions: These findings indicated that auditory short-term training could change human neural activity, suggesting future clinical applications for auditory training.
\end{abstract}

Korean J Audiol 2013;17:105-110

KEY WORDS: Oddball paradigm · Mismatch negativity (MMN) · Auditory short-term training.

\section{Introduction}

Neural plasticity, one of the inherent characteristics of the central nervous system, defines a changing pattern associated with neural form and its connections-based behavioral and learning experience through life. ${ }^{1-3)}$ In the human central auditory system, plasticity is altered by auditory training through repetitive listening tasks over a period of time. ${ }^{4)}$

Meanwhile, electrical measures from the auditory nervous system, called auditory evoked potentials (AEPs), are increasingly used to study dynamic brain activity that subserves auditory perceptual and cognitive processes. AEPs are becoming more popular due to the use of noninvasive techniquesnamely, surface electrodes. Among many AEP measurements, a passive oddball paradigm [i.e., deviant (or oddball) stimuli

This is an Open Access article distributed under the terms of the Creative Commons Attribution Non-Commercial License (http://creativecommons. org/licenses/by-nc/3.0/) which permits unrestricted non-commercial use, distribution, and reproduction in any medium, provided the original work is properly cited. embedded in a sequence of homogenous stimuli] designated as mismatch negativity (MMN) has been used for evaluating auditory training effects in the research field.

In 1995, Kraus, et al. ${ }^{5)}$ examined whether listeners discriminate between two speech-contrasts having different onset frequencies of the second and third formant transitions. After one week of training, the results for 13 normal-hearing listeners indicated that MMN latency and area were significantly changed when compared to before the training. The researchers concluded that these changes indicated experience-related plasticity in central auditory processing by complex signals such as speech. In another study, Tremblay, et al. ${ }^{6}$ trained 10 listeners with normal hearing for one hour per day over 10 days, while asking to discriminate voice-onset time (VOT) difference between $/ \mathrm{mba} /$ and $/ \mathrm{ba} /$ stimuli. After the 10 days' training, the results showed that participants' behavioral scores and N1-P2 amplitude were increased. The authors hypothesized that the increment in N1-P2 amplitude could be considered to reflect crucial neural plasticity associated with improved speech 
perception. ${ }^{6}$ Although most prior studies showed that auditory training for approximately $7-10$ days could change neural activity, recent studies reported a positive relationship between a brief training and neural coding of short-term memory. Other researchers also supported effects of the short-term training, while finding that neurophysiologic changes were observed even from the first day of the 10-day auditory training period when asking to differentiate between two stimuli having different VOTs. They might explain these changes as differences in "rate of learning". 7) That is, the short-term memory could be activated in as little as 30 seconds, resulting in significant changes in brain activity in frontal and parietal cortex and basal ganglia, as well as changes in dopamine receptor density. ${ }^{8)}$

More recently, many contemporary researchers investigated changes in amplitude and latency of event related potential (ERP) during rapid perceptual learning. That is, the rapid improvements in performance within 1-hour training session called 'fast perceptual learning', using two consonant vowel syllables that differed in voice onset time. The MMN results of the training-induced changes showed an increased negative peak while indicating a top-down nonspecific attention effect on neural activity during learning which is coincident with behavioral improvements in speech identification. ${ }^{9)}$ Ben-David, et al. ${ }^{10)}$ also supported a good relationship between rapid physiological changes (i.e., N1 and P2 waves) and learning after 1-hour training by speech identification practice using two synthetic / ba/ and /mba/. Although scalp-recorded ERPs have been instrumental in identifying physiological correlates of auditory perceptual learning, ${ }^{11-13)}$ it is not yet certain that the short-term auditory training can be enough to cause any neural changes in the human central auditory system. Ultimately, the answer will help people who suffer from hearing loss in terms of relevant timeframe for the auditory training and pro- vide them with time-based milestones for training effects.

The purpose of the present study is to evaluate the effects of short-term training using two novel stimuli in normal-hearing adults, by means of behavioral and electrophysiological methods. We hypothesize that the results of the MMN would be significantly changed in terms of latency and amplitude after normal-hearing listeners train to discriminate between two novel stimuli with acoustical similarities for 20 minutes, compared to before the training and to an age-matched control group.

\section{Subjects and Methods}

\section{Subjects}

A total of 20 ( 5 male and 15 female) participants between the ages of 22 and 27 (mean: 24 years old) were randomly recruited. The participants reported a negative history of head or neck abnormalities, ear surgery, otologic disease, head trauma, or head injury. They did not have attention or learning disabilities. They also passed normal criteria of the hearing screening to ensure A-type of tympanogram, the threshold of $15 \mathrm{~dB}$ $\mathrm{HL}$ or better in each ear from 250 to $8000 \mathrm{~Hz}$, and air-bone gaps no greater than $5 \mathrm{~dB}$ HL. They were all right-handed and native Korean speakers. Among the 20 participants, 10 were randomly placed into an experimental group (or training group) whereas the other participants were assigned to a control group. All participants completed the informed consent form.

\section{Stimuli}

To find stimuli in which most normal-hearing listeners were hard to discriminate each other, two synthetic consonant-vowel continua representing the $/ \mathrm{su} /-/ \int \mathrm{u} /$ contrast from a previous study were used, ${ }^{14)}$ which was created by a formant synthesizer. ${ }^{15)}$ The Fig. 1A displays the percentage correct of /su/
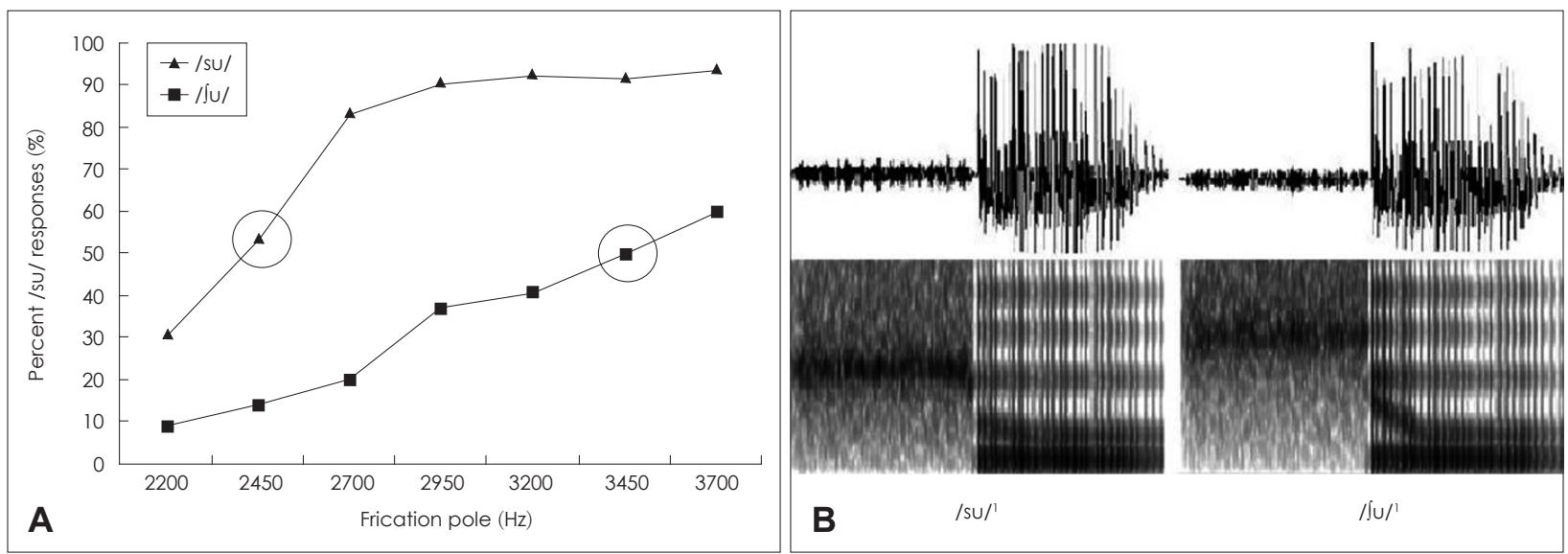

Fig. 1. Two stimuli selected at the level of $50 \%$ correct response as a function of pole frequency of the frication spectrum (A)(Lee and Banhg, 2012); /su/ ${ }^{1}$ stimulus spectrogram with 2450 pole frequency and $1200 \mathrm{~Hz}$ transition and /Ju/ ${ }^{1}$ stimulus spectrogram with 3450 pole frequency and $1800 \mathrm{~Hz}$ transition (B). 
and $/ \int \mathrm{u} /$ as a function of pole frequency spectrum. We selected two stimuli at 50 percent correct of the $/ \mathrm{su} /$ and $/ \int \mathrm{u} / \mathrm{continu}-$ um. They were /su/ stimulus, which has pole frequency with $2450 \mathrm{~Hz}$ and transition signal with $1200 \mathrm{~Hz}$, and / $\mathrm{J}$ / stimulus, which has pole frequency with $3450 \mathrm{~Hz}$ and transition signal with $1800 \mathrm{~Hz}$ (Fig. 1B). We decided to call these selected stim$\mathrm{uli} / \mathrm{su} /{ }^{1}$ and $/ \mathrm{Ju}_{\mathrm{u}}{ }^{1}$ in this study.

In the MMN parameter, each stimulus had $500 \mathrm{msec}$ in duration and inter stimulus interval was $1000 \mathrm{msec}$. Sampling frequency and sampling bit were adjusted to $48000 \mathrm{~Hz}$ and 16 bit, respectively. Root mean square was also adjusted. The stimuli were presented using an oddball paradigm in which the deviant stimulus $/ \int \mathrm{u} /{ }^{1}$ was presented in a series of standard stimuli $/ \mathrm{su} /{ }^{1}$ with the ratio of $1: 5$. Stimulus intensity level was $75 \mathrm{~dB}$ nHL and stimulus interval was $1.1 \mathrm{sec}$. Responses were elicited by approximately 350 stimuli. Evoked recordings were filtered from 1 to $100 \mathrm{~Hz}$. Responses were amplified with a gain of 50000 and the recording window included 0 to 500 msec time. Artifacts were rejected during the test if located at $95 \mathrm{mV}$ or above. Two channel-electrodes were placed at $\mathrm{Cz}$ as reference and at $\mathrm{A} 1$ and $\mathrm{A} 2$ as active and ground, respectively (Bio-Logic Navigator Pro system; Bio-Logic Co., Willow Hill, PA, USA), Stimuli were presented to the right ear by an inserted earphone.

\section{Experimental procedures}

For the experimental group, first of all, MMN and behavioral discrimination tests were conducted as a baseline for the study. The behavioral discrimination test, which consists of 20 trials of $/ \mathrm{su} /{ }^{1}$ and $/ \int \mathrm{u} /{ }^{1}$ stimuli, was presented through a computer speaker at the most comfortable level for each subject and the result scores were rated from 0 to 100 percent. During the short-term auditory training for 20 minutes, the subjects were instructed to respond that two stimuli were either same or different with feedback and also to respond whether the stimulus was $/ \mathrm{su} /{ }^{1}$ or $/ \int \mathrm{u} /{ }^{1}$ by an alternative forced-choice task. After the 20-min training session, each subject participated in the second set of MMN and behavioral tests.

On the other hand, the control group also conducted the first MMN test but did not undergo the behavioral test. Subjects in the control group were asked to read an easy book instead of doing the 20-min training. Then, the second MMN test was assessed.

\section{Data analysis}

Pre-training MMN results for the two groups were compared using the independent t-test. For each of the experimental and control groups, the paired t-test was used to compare the MMN results between pre-training (first) and post-training (second). In the experimental group, the behavioral test was also analyzed with the paired t-test to determine the effect of training. A Holm's Bonferroni procedure was used to control for Type I error associated multiple testing. A family wise error rate of 0.05 was adopted. A software for the statistical analysis used SPSS (ver.20, IBM Co.).

\section{Results}

\section{Training effect on the behavioral test}

In the experimental group, the discrimination scores before the training (or pre-training) ranged from 20 to 65 percent correct (mean=42 percent), whereas those after the training (or post-training) ranged from 60 to 100 percent correct (mean= 92 percent). The ability to discriminate $/ \mathrm{su} /{ }^{1}$ and $/ \int \mathrm{u} /{ }^{1}$ improved significantly after training $[\mathrm{t}(9)=-6.64, p=0.000]$.

\section{Training effect in electrophysiological results}

To determine whether the experimental and the control groups were similar in terms of baseline MMN results, the independent t-test was conducted. The results showed that baseline MMN onset latency, duration, and area were not significantly different between the group [onset latency: $\mathrm{t}(18)=0.12$, $p=0.907$; duration: $\mathrm{t}(13.5)=-0.81, p=0.432$; area: $\mathrm{t}(18)=-0.41$, $p=0.688]$ (Fig. 2).

MMN results before and after the training were compared using the paired t-test. As shown in Fig. 2A, MMN onset latency significantly decreased in the experimental group $[\mathrm{t}$ $(9)=4.49$, adjusted $p=0.005$ ], but the control group showed no significant differences in onset latency between before and after the training $[\mathrm{t}(9)=-2.03$, adjusted $p=0.011]$. The MMN duration was significantly increased in the experimental group after the training when compared to before the training $[\mathrm{t}(9)=-4.34$, adjusted $p=0.004]$, but the results of two tests for the control group were similar $[\mathrm{t}(9)=1.39$, adjusted $p=0.216]$ (Fig. 2B). An increase in MMN area was also significant in the experimental group [ $\mathrm{t}(9)=-3.20, p=0.005]$, but not in the control group [ $\mathrm{t}(9)=-0.68$, adjusted $p=0.396]$ (Fig. 2C).

After training of the experimental group, waveform morphology of MMN became wider than that before the training (Fig. 3A). However, two MMN morphologies of the control group were quite the same (Fig. 3B).

\section{Discussion}

The aim of the present study was to investigate any neural changes after short-term auditory training in normal-hearing adults. We found significant changes in both behavioral and 

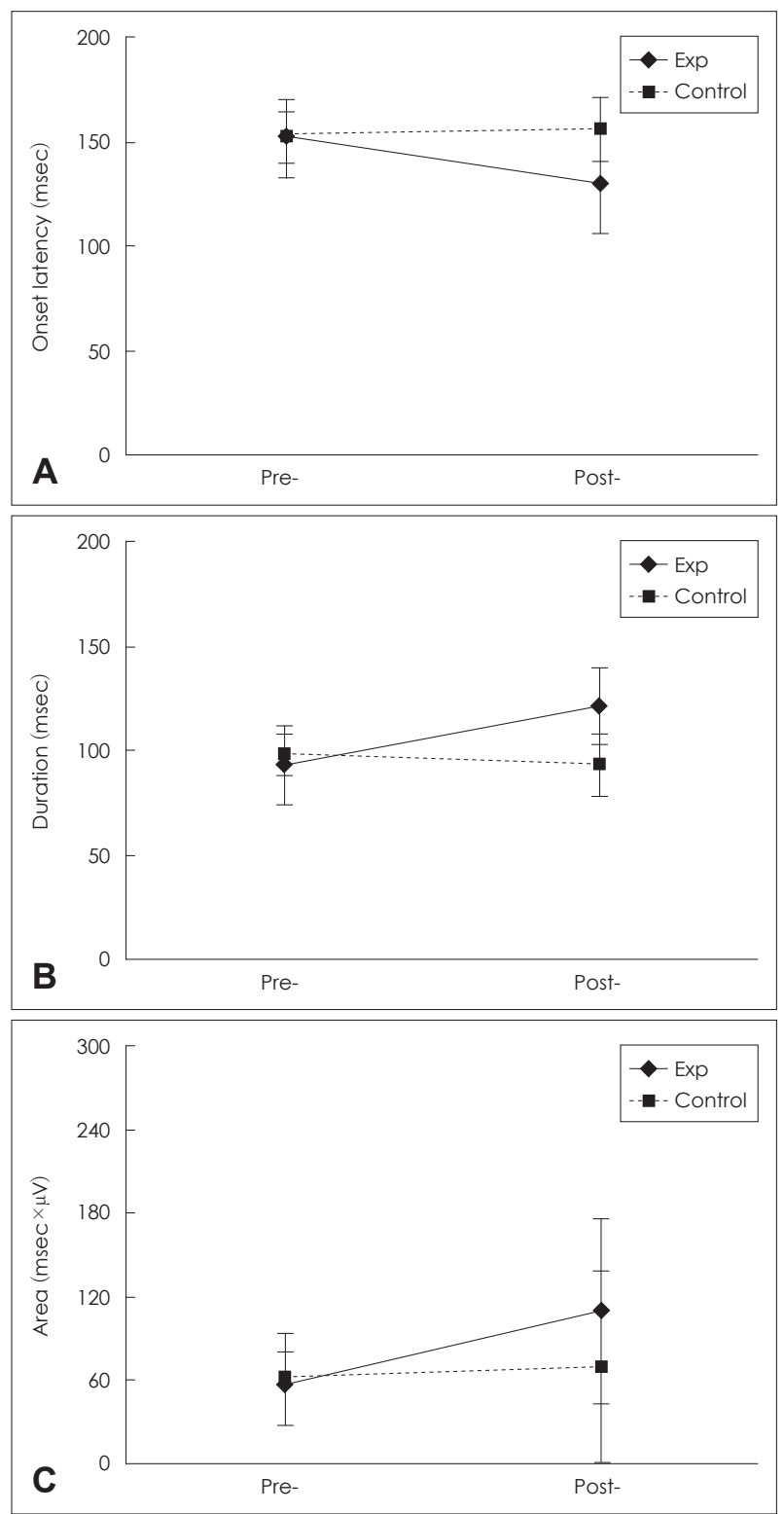

Fig. 2. Tendencies of main elements of MMN results in the experimental group (solid line) and the control group (dotted line). Each panel represents $\mathrm{MMN}$ onset $(\mathrm{A})$, duration $(\mathrm{B})$, and area $(\mathrm{C})$. Pre- means pre-auditory training, and post- means post-auditory training. Error bars indicate standard deviation. MMN: mismatch negativity.

electrophysiological results. The discrimination scores of the behavioral test were significantly increased. In the electrophysiological test, onset latency, duration, and area of MMN were significantly changed following the short-term training. These findings supported the results of previous research. Tremblay, et al. ${ }^{7)}$ reported that onset latency, duration, and area of MMN were also significantly changed when normal-hearing adults were trained to discriminate and identify VOT varying from $-50 \mathrm{~ms}$ to $+50 \mathrm{~ms}$ in labial and alveolar continua. Also, a study by Kraus, et al. ${ }^{5)}$ revealed the same results when subjects were trained to discriminate between two speech-contrast stimuli $\left(/ \mathrm{da} /{ }^{1}\right.$ and $/ \mathrm{da} /{ }^{2}$, which were different in the onset frequencies of the second and third formant transitions). They concluded that the pre-attentive central nervous system underlying perceptual learning would be altered through auditory training. ${ }^{16)}$

In this study, a very short period of training (i.e., $20 \mathrm{~min}$ ) was conducted. The MMN results showed that the onset latency was shown earlier and the area increased about double when compared to the pre-training condition. According to previous studies, subjects with higher discrimination ability showed shorter latency, which is a faster response time and increased amplitude/area, and the activation of the neural generators was enhanced by training. ${ }^{6,17)}$ Thus, in the present study, the faster onset time and the increased area could show the neural change that had occurred even after only 20-min shortterm training. Intensive training in the short term could improve not only the discrimination ability of the listeners but also change the neural activity of speech sounds. It follows, therefore, that we should not overlook even brief training. However, our study did not determine whether the training effects were retained over time. This needs to be explored in future studies.

\section{Relationship between stimulus characteristics and short-term training effects}

The two novel stimuli used have two different cues in terms of the pole of frequency spectrum and the formant transition cues. ${ }^{14)}$ As a result, behavioral scores for the discrimination task were 42 percent before the training and reached 92 percent after the training. When taking into account that the training was conducted for only 20 minutes, an approximately 50 percent increase deserves to be considered. On the contrary, previous research reported an increase of 36 percent in Tremblay, et al.'s ${ }^{16)}$ study and an improvement of 22 percent in another Tremblay, et al.'s ${ }^{6)}$ study, when listeners were trained for 7 to 9 days. Although they had a longer duration of training, lesser improvement of the scores was reported than in our study. One reason for the discrepancy was stimulus characteristic. Gottselig, et al. ${ }^{13)}$ found that the difficulty in training novel stimuli affected MMN results. They indicated high correlations between learning and changes in MMN when subjects performed the easier discrimination. On the other hand, for the difficult discrimination task, the MMN change was not statistically significant. They suggested that the more difficult tasks might need longer training periods.

Moreover, each stimulus has different cues and these cues can affect the difficulty of the training to discriminate two stimuli. Previous studies ${ }^{6,16)}$ used two stimuli that have one cue, different VOT (/ba/ has $-10 \mathrm{msec}$ VOT and $/ \mathrm{mba} /$ has $-20 \mathrm{msec}$ VOT). However, the present study used two stimuli that have 
Fig. 3. Grand average of waveform morphology before the training (or pretraining; upper wave) and after the training (or post-training; lower wave) in each group ( $A$ for the experimental group and B for the control group).
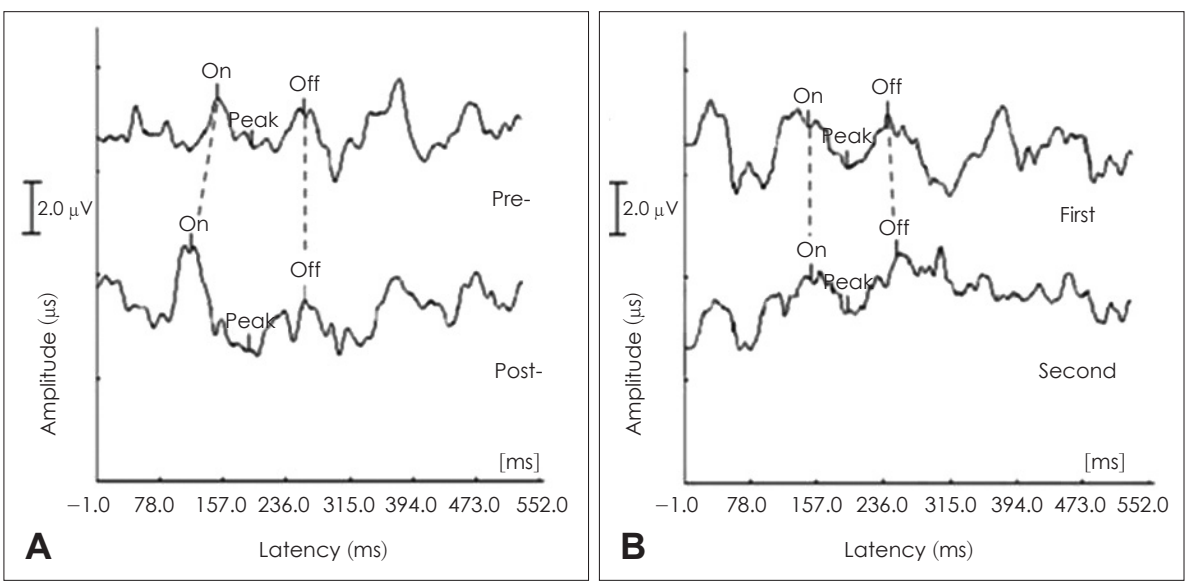

two cues, different pole frequency and transition cues. These might help listeners discriminate between two stimuli more easily during the training. This is why the behavioral scores after the training showed a greater increase than the two previous studies despite the relatively short time period. Consequently, stimulus characteristics can affect not only the difficulty of the training but also the after-training results.

\section{Clinical application and further study}

The present study confirmed that intensive and repeated auditory training for the short term resulted in changes in MMN as well as behavioral discrimination ability. This may prove that changes in neural activity occur rapidly during the training. Therefore, this can be a useful tool to apply for the confirmation of auditory training effects. The training-induced changes transfer trained stimuli to other auditory stimuli that were not used during the training sessions, called "transfer of learning". ${ }^{16)}$ This generalization is what audiologists expect as the ultimate goal of auditory training. When the training-induced changes transfer to new listening situations, training effects could prove successful. In the related study by Tremblay, et al., ${ }^{16)}$ nine normal-hearing listeners were trained to identify a $/ \mathrm{mba} /$ stimulus that had $-20 \mathrm{msec}$ VOT. After a five-day training session, the effects of training not only resulted in improvement of the ability in both behavioral and electrophysiological tests, but also transferred to the $-20 \mathrm{msec}$ VOT /nda/ stimulus that was not trained. If the present study also found "transfer of learning," the importance of short-term training is well worth considering. Therefore, further research is needed to examine whether short-term training effects transfer to other stimuli in categories (varied pole frequency and transition cue) when subjects were only trained to discriminate $/ \mathrm{su} /{ }^{1}$ and $/ \int \mathrm{u} /{ }^{1}$ stimuli.

AEPs including MMN have been proven to reflect training effects not only in normal-hearing listeners but also in hearing aid and cochlear implant users. ${ }^{18)}$ In this study, only young, nor- mal-hearing listeners participated. Because of their healthy auditory systems, very brief auditory training was effective in changing their neural activity. However, short-term auditory training might not affect neural activity changes in hearing-impaired listeners due to their abnormal auditory systems. Research should be conducted to determine whether the same results occur with hearing aid and cochlear implant users under short-term training. Also, the contribution of amplification in AEP recording ${ }^{19)}$ remains poorly understood. In the future studies, the relationship between device characteristics and AEP responses should be determined.

\section{Conclusion}

This study examined whether short-term auditory training of 20 minutes can result in neural changes. After the short-term training, behavioral scores significantly increased, and all the MMN results (MMN onset latency, duration, area) were also significantly changed. These results indicate that short-term auditory training may be enough to change neural activity, suggesting clinical implications for short-term training.

\section{Acknowledgments}

We would like to thank Dr. Mark Hedrick (Univ. of Tennessee) for generating the experimental stimuli.

\section{REFERENCES}

1) Eggermont JJ. The correlative brain: theory and experiment in neural interaction. In: Fristion KJ, editor. Studies of Brain Function. New York: Springer verlag;1990. p.1-36.

2) Gilbert CD, Sigman M, Crist RE. The neural basis of perceptual learning. Neuron 2001;31:681-97.

3) Rubel E. Ontogeny of structure and function in the vertebrate auditory system. In: Jackson M, editor. Handbook of Sensory Systems. New York: Springer verlag;1978. p.135-237.

4) Tremblay KL. Training-related changes in the brain: evidence from human auditory-evoked potentials. Semin Hear 2007;28:120-32.

5) Kraus N, McGee T, Carrell TD, King C, Tremblay K, Nico T. Cen- 
tral auditory system plasticity associated with speech discrimination training. J Cognitive Neurosci 1995;7:25-32.

6) Tremblay K, Kraus N, McGee T, Ponton C, Otis B. Central auditory plasticity: changes in the N1-P2 complex after speech-sound training. Ear Hear 2001;22:79-90.

7) Tremblay K, Kraus N, McGee T. The time course of auditory perceptual learning: neurophysiological changes during speech-sound training. Neuroreport 1998;9:3557-60.

8) Klingberg T. Training and plasticity of working memory. Trends Cogn Sci 2010;14:317-24.

9) Alain C, Campeanu S, Tremblay K. Changes in sensory evoked responses coincide with rapid improvement in speech identification performance. J Cogn Neurosci 2010;22:392-403.

10) Ben-David BM, Campeanu S, Tremblay KL, Alain C. Auditory evoked potentials dissociate rapid perceptual learning from task repetition without learning. Psychophysiology 2011;48:797-807.

11) Atienza M, Cantero JL, Dominguez-Marin E. The time course of neural changes underlying auditory perceptual learning. Learn Mem 2002;9:138-50.

12) Brattico E, Tervaniemi M, Picton TW. Effects of brief discrimina- tion-training on the auditory N1 wave. Neuroreport 2003;14:2489-92.

13) Gottselig JM, Brandeis D, Hofer-Tinguely G, Borbély AA, Achermann P. Human central auditory plasticity associated with tone sequence learning. Learn Mem 2004;11:162-71.

14) Lee S, Banhg J. Cue weighting strategy of fricative perception in noise for normal hearing listeners. Audiology 2012;8:52-60.

15) Klatt DH. Software for a cascade/parallel formant synthesizer. J Acoust Soc Am 1980;67:971-95.

16) Tremblay K, Kraus N, Carrell TD, McGee T. Central auditory system plasticity: generalization to novel stimuli following listening training. J Acoust Soc Am 1997;102:3762-73.

17) Beauchamp CM, Stelmack RM. The chronometry of mental ability: an event-related potential analysis of an auditory oddball discrimination task. Intelligence 2006;34:571-86.

18) Korczak PA, Kurtzberg D, Stapells DR. Effects of sensorineural hearing loss and personal hearing AIDS on cortical event-related potential and behavioral measures of speech-sound processing. Ear Hear 2005;26:165-85.

19) Martin BA, Tremblay KL, Korczak P. Speech evoked potentials: from the laboratory to the clinic. Ear Hear 2008;29:285-313. 